\title{
The Swift satellite lives up to its name, revealing cosmic explosions as they happen
}

\author{
By RhaAna L.C. Starling \\ University of Leicester, Dept. of Physics and Astronomy, Leicester LE1 7RH, UK
}

Gamma-ray Bursts are the most powerful objects in the Universe. Discovered in the 1960's as brief flashes of gamma-radiation, we now know they emit across the entire electromagnetic spectrum, are located in distant galaxies and comprise two distinct populations, one of which may originate in the deaths of massive stars. The launch of the Swift satellite in 2004 has brought a flurry of new discoveries, advancing our understanding of these sources and the galaxies that host them. We highlight a number of important results from the Swift era thus far.

Keywords: gamma-rays: bursts - massive stars - supernovae - galaxies

\section{Introduction}

(a) What are Gamma-ray Bursts?

The old saying 'the early bird catches the worm' is never more true than when studying the most powerful explosions in the Universe - Gamma-ray Bursts. These monster events - second only to the Big Bang in energy release - are occurring every day all across the Universe, and were discovered accidentally during the cold war by spy satellites looking for the tell-tale signs of nuclear weapons testing (Klebesadel et al. 1973). The bursts of gamma-rays they detected seemed to be coming not from the Earth but from space and galaxies beyond our own (later confirmed: Paczyński 1995; Metzger 1997).

Decades on, astronomers have identified the origins of perhaps three quarters of the Gamma-ray Burst (GRB) population: the so-called long GRBs with gammaray durations of more than a couple of seconds. They emerge from the deaths of very massive stars, where a black hole and a supernova are formed (see Section 4), together with two highly relativistic jets, one of which points directly at us. GRBs are the ultimate probes of extreme physical processes which cannot be simulated in any laboratory on Earth. We observe the radiation created by shocks in the jet at gamma- and X-ray wavelengths, lasting a few to a few hundred seconds and termed the prompt emission. The innermost region where the powerful jets are launched is known as the central engine (see Section 2). The prompt emission is followed by the so-called afterglow emission after the jet breaks out of the star and emits at longer wavelengths down through the optical and radio bands. It is this afterglow, fading to nothing on timescales of days to months, that has been the main catalyst for our understanding of GRBs.

But the hunt for GRB origins is not over. About a quarter of all detected GRBs exist only for a fraction of a second, seen as a single short spike against 
the gamma-ray background. Blink, and you have missed it! These sources, known as short GRBs, are also preferentially seen in the higher energy bands making them spectrally harder than the long bursts (Fig. 2, e.g. Kouveliotou et al. 1993). The afterglows of this class of GRBs remained elusive for decades; their eventual discovery is described in Section 3.

GRBs are bright and hence can be seen out to great distances. They allow us to probe the furthest reaches of the Universe with look-back times currently stretching to 13 gigayears (e.g. Kawai et al. 2006). Section 5 explains their potential as very powerful tools to study the very first stars, how galaxies were assembled and how they have evolved to the present day.

\section{(b) Introducing the Swift satellite}

The Swift satellite (Gehrels et al. 2004), launched into low-Earth orbit in November 2004, detects GRBs at a rate of $\sim 100$ per year. It carries 3 instruments: a widefield gamma-ray telescope named BAT (Burst Alert Telescope), an X-ray telescope named XRT and an optical and ultraviolet telescope named UVOT. When a flash of gamma-rays is detected with the BAT the spacecraft then quickly turns itself towards the GRB (slews) to begin on-board X-ray and optical/ultraviolet(UV) observations typically $70-100$ seconds later. The GRB location on the sky is sent out automatically and can be picked up by ground-based observers. The first data are also relayed to Earth in near real-time and a number of important characteristics of the object can be measured allowing informed decisions to be made on how to proceed with follow-up observations. In this game the ability to turn the spacecraft around incredibly quickly (within minutes) is key to detecting the rapidly fading afterglows and therefore locating the GRB to high precision.

Swift's unique fast-slew capability, unprecedented sensitivity, large field of view for GRB detection together with simultaneous coverage of several crucial wavelength regimes and the dedicated fast data-downlink system provides a wealth of detail on how these sources behave with time (through light curves) and with wavelength (through spectra).

\section{Probing extreme physical processes}

The intrinsic luminosities of GRBs are immense, producing as much energy in tens of seconds as the Sun will emit in its entire lifetime. If this energy is emitted in all directions plausible progenitor models are stretched to the limit (in some cases energies are in excess of $10^{53} \mathrm{erg}$ ) and so the radiation must be confined to a cone or jet. While the progenitors of short and long-duration bursts are clearly different, the mechanisms producing the gamma-ray burst and subsequent afterglow are similar. In both cases we are witnessing a blast wave propagating out from a central object, in which shocks create both the initial gamma-ray emission and the broad-band afterglow emission. The overall behaviour of the blast wave can be represented by a set of power laws characteristic of synchrotron emission generated by the acceleration of fast-moving particles in magnetic fields in the shock front (for a review see e.g. Zhang \& Mészáros 2004). The physical parameters can therefore be probed by observations with good temporal and spectral coverage - requiring a dedicated space mission.

Article submitted to Royal Society 
No two GRBs look the same, but there are features common to many of the X-ray afterglows (Fig. 2, Nousek et al. 2006; O'Brien et al. 2006). After the initial prompt phase the canonical afterglow decays very steeply before flattening out to what is known as a plateau phase. The light curve then breaks to the steeper decay rate known from pre-Swift data and may steepen again at a day or so after the GRB began indicating the sideways spreading of the jet as the blast wave decelerates (Rhoads 1997). Swift has really uncovered the timezone in which the observed emission transitions from prompt GRB dominated to afterglow dominated.

Striking features - sharp peaks superposed on the steadily fading light - can be seen in around half of all Swift GRB X-ray light curves, and are called Xray flares (e.g. Burrows et al. 2005). X-ray flares can be very intense - in one case a giant flare was observed which contained almost as much energy as the GRB itself (GRB 050502B, Falcone et al. 2005). This requires that through some process additional energy is injected into the blast wave. Further indications of the continuation of the central engine arise in particularly long-lasting GRB prompt emission continuing for hundreds of seconds (e.g. GRB 070616, Starling et al. 2008) and lengthy afterglows, such as GRB 060729 which was observable in the X-rays for 125 days, far longer than any other GRB afterglow (Grupe et al. 2007). Energy is thought to have been re-injected into the moving blast wave or shock front in order for the afterglow to be bright for such a long time. Emission from jets is seen in various forms from many types of objects all across the Universe, from active galaxies to X-ray binaries to young stellar objects, where the GRB jets clearly have the fastest motion approaching the speed of light. These new revelations in GRB science are bringing us closer to the central engine, allowing studies of fundamental processes such as acceleration mechanisms in shocks, jet collimation and magnetic field generation in shock fronts.

In a few particularly bright individual cases the jet structure and physics of the internal engine have been revealed like never before. GRB 080319B was the second of four GRBs to go off on 19th March 2008. This was truly the monster of all bursts: the brightest from optical to X-rays yet seen. The source briefly reached a visual magnitude of 5.3, meaning that if you happened to be looking up at the right time and place you would have seen it with the naked eye! The light would be coming to you from redshift 1 , equivalent to $\sim 7.5$ gigayears ago, demonstrating the sheer power in these events. The dataset collected for this GRB is arguably the most complete to date, and efforts are now underway to understand the workings of the central engine (e.g. Racusin et al. 2008).

\section{The discovery of Short burst afterglows}

Progress in understanding the very shortest duration Gamma-ray Bursts, lasting less than 2 seconds in the high energy gamma-rays, has lagged far behind long burst studies. This was almost entirely due to the lack of significant detections of these sources using X-ray and optical telescopes. The most ground-breaking discovery made with the Swift satellite to date is undoubtedly the first detection of afterglow emission from any short burst. The location of the faint (just 11 source photons) X-ray afterglow of GRB 050509B (Gehrels et al. 2005) was good to four arcseconds (error circle radius), and whilst no optical counterpart was identified, the local neighbourhood of the burst provided some clues as to the GRB progeni- 


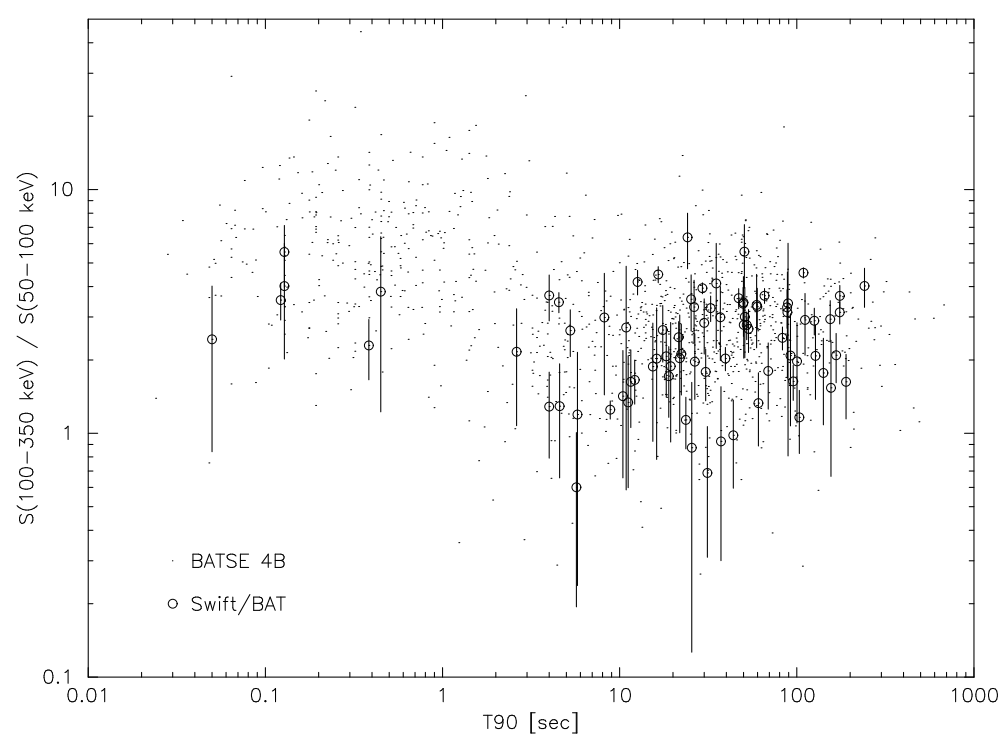

Figure 1. Hardness-duration diagram for Swift bursts up to 2006 (data points with error bars) overlaid on the sample from Swift's predecessor the Burst And Transient Source Experiment. A bimodality can be seen in the GRB population. From Sakamoto et al. (2006).

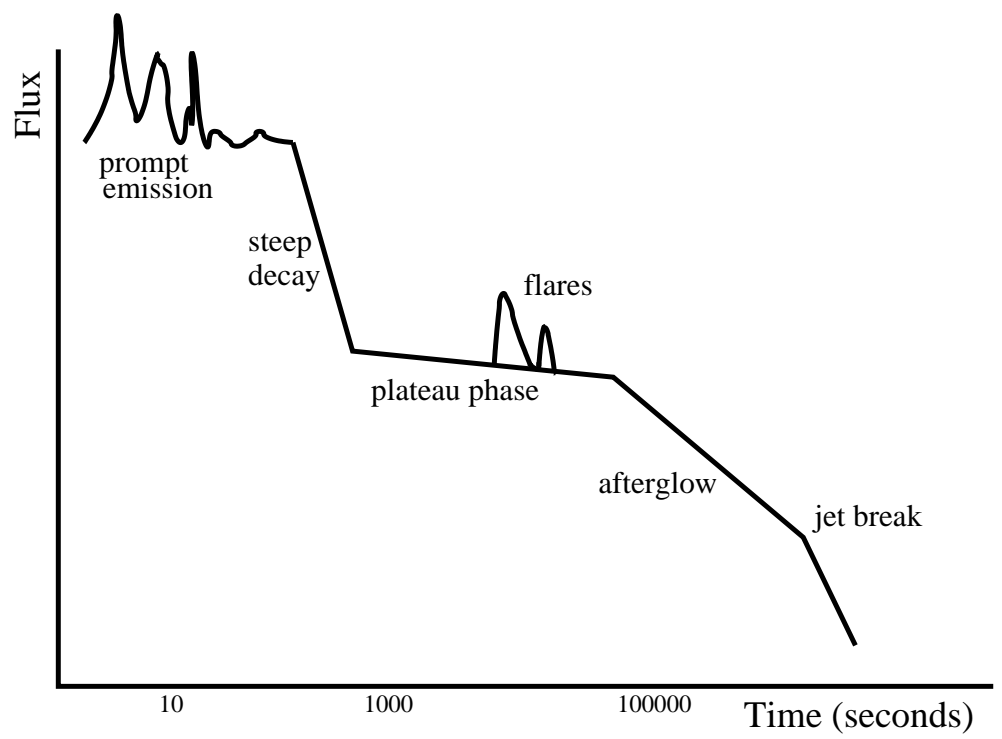

Figure 2. Sketch of the canonical Swift X-ray light curve.

tor. Within the Swift X-ray position error circle a nearby (redshift $z=0.225$ ) galaxy cluster comprising a large elliptical and several smaller galaxies was seen through essential follow-up by ground-based telescopes including the Very Large Telescope in Chile and Keck in Hawaii (Fig. 3, e.g. Hjorth et al. 2005). The chance coincidence of a GRB with the large elliptical is very low, suggesting this old red galaxy, no longer forming stars, may have hosted the GRB. Searches for the rise of an accom- 
panying supernova component (expected for nearby long GRBs) found nothing to stringent limits, and together with the elliptical host galaxy this provided evidence in support of an origin for short bursts which is completely different from the massive star origins of their long-duration cousins: the compact binary merger scenario. This scenario was proposed for the origin of short-duration GRBs many years ago and works by allowing the orbits of the two stars to decay through gravitational inspiral, bringing them gradually closer together with time eventually to merge (see e.g. Nakar 2007 for a review). Given that a large fraction of stars in our Universe are thought to exist in pairs, orbiting each other in a binary system, a double-star merger seems like a disaster waiting to happen in every corner of the Universe. However for a powerful GRB to be produced these stars must be extremely small and dense and these systems are probably rare. Following the successful detection of an X-ray afterglow from short GRB 050509B came the detection of an optical afterglow for the short GRB 050709 (Hjorth et al. 2005b; Fox et al. 2005). The subarcsecond position of the optical afterglow led to successful identification of the host galaxy, lying at a distance $z=0.16$. Thereafter several more afterglow detections were made at both X-ray and optical wavelengths. The sample shows that while short GRB afterglows are generally less distant, their afterglows are intrinsically fainter than the long GRBs but follow the same decays and spectral shapes caused by synchrotron emission in a decelerating blast wave. There appears to be a tendency towards associations with late-type (elliptical) galaxies at $z<1$ with very little or no current star formation. If these bursts originate in a binary merger (the currently favoured model) we might expect to see them more frequently in low density environments, kicked out of the denser regions in which they were born during the earlier supernova phase. This is clearly in contrast to the highly star-forming regions in which we expect the long bursts to occur. There are notable exceptions to this rule however, and while the sample size is still small a complete picture is difficult to form.

Astronomers could recall a small number of similar short duration bursts of gamma-rays first seen back in 1979, which caused quite a stir. These gamma-ray flashes come from our own galaxy and are not gamma-ray bursts as we know today, but are in fact flares from so-called soft gamma repeaters or SGRs. Few such objects are known, and are thought to be neutron stars with incredibly strong magnetic fields, also known as magnetars, on which a 'star-quake' may trigger intense flaring activity. In 2004 a giant flare erupted from known Galactic magnetar SGR 1806-20 (Palmer et al. 2005) prompting the question: if such a flare were to go off in another galaxy would we see it? The answer is yes, it could have been seen out to tens of megaparsecs in nearby galaxies, and would then be difficult to distinguish from a short GRB. Comparison of the locations of short GRBs and local galaxies led to a positive correlation for $10-25 \%$ of the sample, suggesting that this fraction of apparent short duration GRBs may in fact come from SGR flares in nearby galaxies (Tanvir et al. 2005).

While hard proof of the origins of short GRBs still lies at arm's reach, we now know that there is more than a single progenitor for these brief high energy flashes, and current and future technology affords more opportunities to go after their faint afterglows. 


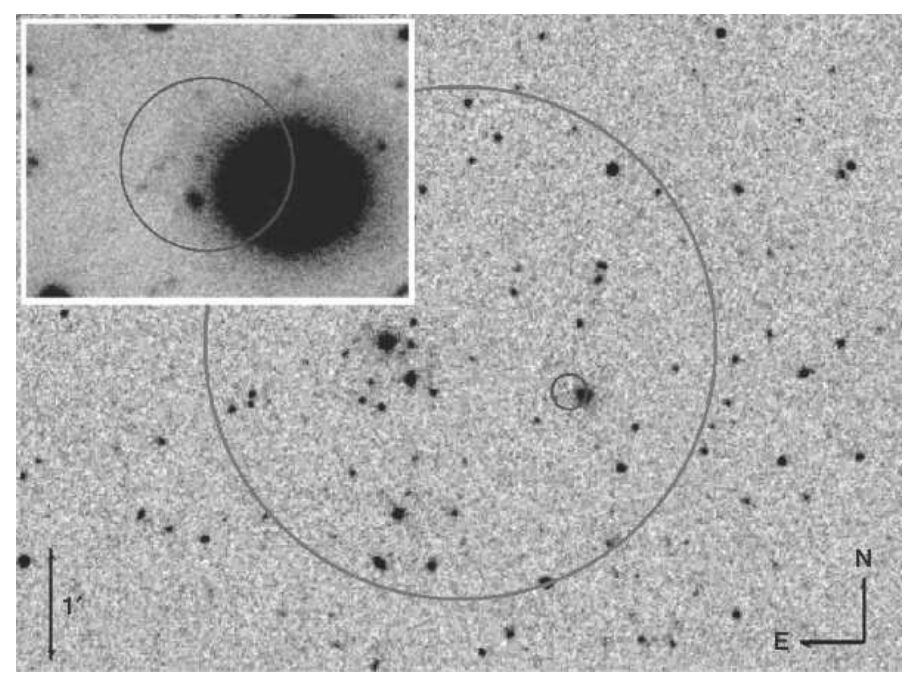

Figure 3. A digitzed sky survey image shows the localisation of the first short GRB afterglow in X-rays (small circle; large circle indicates the gamma-ray position). In the inset is a close-up of the region imaged with the Very Large Telescope showing the association of the burst with a large elliptical galaxy. From Gehrels et al. (2005).

\section{The GRB-supernova connection}

A connection between long-duration GRBs and supernovae has long been established (MacFadyen \& Woosley 1999). The association was made observationally when within the error circle of GRB 980425 a bright and energetic supernova was found (SN1998bw, Galama et al. 1998). Initially this was considered by some a chance coincidence, but in the spectra of subsequent long GRBs further supernova signatures were apparent; most famously in GRB 030329 (Hjorth et al. 2003; Stanek et al. 2003). This provided proof of the origin of at least some long GRBs in the core collapse of very massive stars which have lost their outer hydrogen layers, allowing the GRB to escape without being smothered by an extended stellar envelope. While all long GRBs could be accompanied by a supernova, the reverse is not true. It is estimated that core collapse supernovae are 1000-10,000 times more frequent than GRBs. GRB-related supernovae have been studied in small samples and are found to be brighter than ordinary supernovae of the same type when scaled to a common distance. The supernova ejecta radiates through radioactive decay, which can rise above the optical afterglow light from the GRB several days after the GRB occurred but is frequently outshone by the afterglow.

A missing piece in this puzzle has been recovered by Swift observations of nearby GRB 060218 and SN2006aj. This source had a particularly weak afterglow allowing a clear view of the supernova signatures. Not only was the radioactive non-thermal emission bump seen in the optical, but a thermal emission bump was also apparent, moving from X-ray energies to the lower energy UV band with time. This striking result is interpreted as shock breakout as the supernova emerges from the star (Fig. 4, Campana et al. 2006; Pian et al. 2006; Mazzali et al. 2006). This was the first observation of the onset of a GRB-supernova. In 2008 Swift serendipitously caught an ordinary (non-GRB) supernova in the act of exploding while observing an earlier supernova in the same galaxy. Supernovae are usually discovered by 


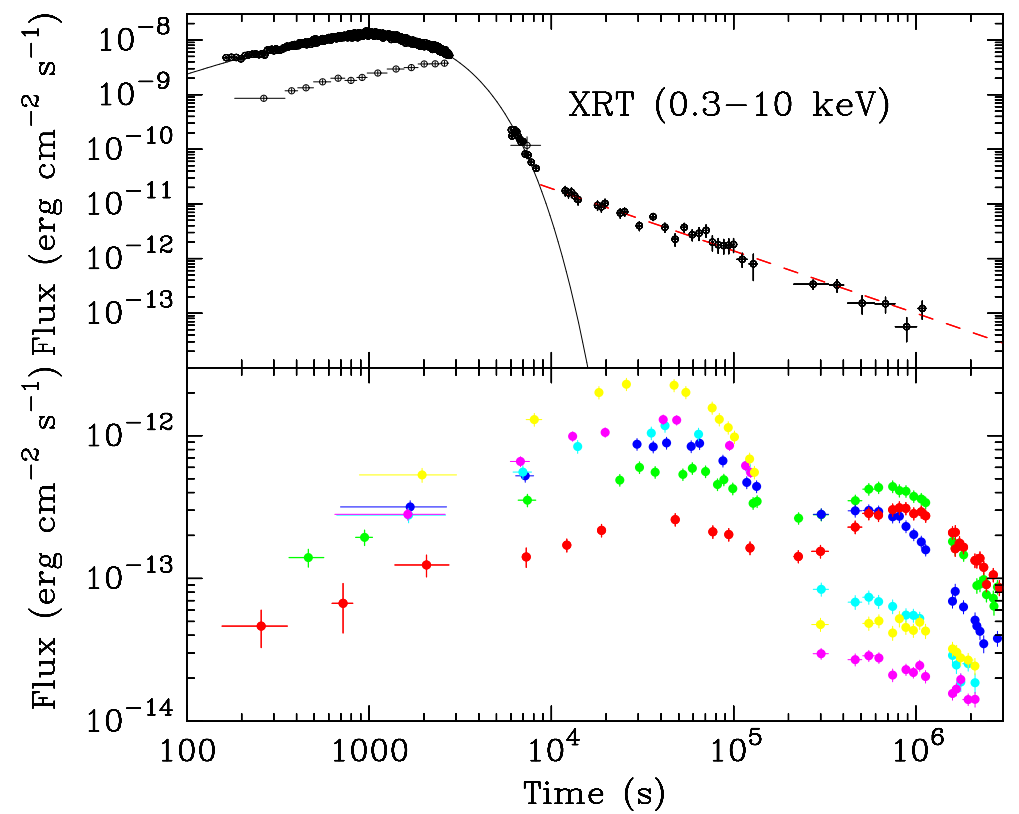

Figure 4. Swift X-ray (top panel) and optical/UV (lower panel) light curves. The bump seen first in X-rays and then in UV is interpreted as early emission from a supernova shock breakout. The later optical bump arises from the radioactive-decay-driven ejecta. From Campana et al. (2006, including colour version of this figure).

the rise in their optical emission from the radioactive decay driven ejecta. This spectacular observation showed an initial spike of X-ray emission right at the onset of the event, again most likely from shock breakout (Soderberg et al. 2008).

While the first signs of core collapse are being discovered, there remain two well studied long GRBs for which no accompanying supernova could be found to very constraining limits (GRBs 060505 and 060614, e.g. Fynbo et al. 2006). Supernovae with no optical signatures were predicted long before the launch of the Swift satellite, and can now be tested with the high quality data available. These two cases perhaps highlight our ignorance of the supernova mechanism, of what type of stars can produce GRBs, or reveal a link from long GRBs to the supernova-less short GRB population.

\section{A glimpse into the very distant Universe}

Swift's first year of operation really did usher GRB science into a new era of discovery. The discovery of the most distant GRB to date, which exploded $13 \mathrm{Gyr}$ ago, came in September 2005. For some time astronomers have been hopeful that GRBs would point us to the very first stars and to conditions in the early Universe at a time when most galaxies had not yet had time to form. As the most luminous objects in the Universe, long GRBs have enormous potential as probes of the early Universe. The bright afterglows act as a backlight illuminating all material between us and the burst. Gas and dust in the host galaxy that lies along the line-of-sight to the GRB absorbs the GRB emission and is imprinted on the afterglow spectrum. This reveals the chemical makeup of the host, and has led to an understanding of 
host galaxies made possible by target-of-opportunity programmes on ground-based telescopes through which GRBs can be observed very shortly after they have gone off. Robotic telescopes can automatically respond to GRB alerts directly from the satellite, and now the same is true of some of the larger world-class telecopes in what is termed Rapid Response Mode. In this mode for example the $8.2 \mathrm{~m}$ Very Large Telescope in Chile can repoint and make observations in just 8 minutes!

Long GRBs tend to lie in small, faint, blue galaxies. The average of the GRB redshift distribution falls at $z=2.3$ (Fig. 5, Jakobsson et al. 2004). Hubble Space Telescope images of a large number of these hosts showed that the GRB commonly lies in the brightest region of the galaxy where star formation is most vigorous (Fruchter et al. 2006). Conditions in the host galaxies are used to feed back into models for long GRB progenitors. For example, afterglow spectroscopy has shown that these galaxies have a lower ratio of metals to hydrogen than does our local neighbourhood in the Milky Way, possibly reaching as metal-poor as one 1/100 the metallicity of the Sun (e.g. Starling et al. 2005). The metal content of a star moderates the outflow of material in its wind, and in turn the properties of the stellar wind are crucial in determining the nature of the stars' final demise (Woosley \& Heger 2005; Yoon \& Langer 2005).

One of the great successes from ground-based telescopes in the fast-reponse era came when astronomers witnessed the dramatic effects that a GRB can have on its environment. GRB 060418 was spectroscopically observed at the Very Large Telescope just 11 minutes after the GRB began. Gas in the host galaxy can be excited by the blast of high energy radiation from the GRB, pushing electrons into higher states in their atoms. The gas then de-excites back to normal levels on a timescale of minutes to hours and these variations have been measured in optical spectral lines (Vreeswijk et al. 2007). The variability as a function of time gives us a measure of the density, temperature and chemical composition of the faint and distant galaxies that host GRBs. We now know that we can locate these galaxies at just $700 \mathrm{Myr}$ after the Big Bang (Kawai et al. 2005). Even at these imponderable look-back times the bright afterglow, its light shifted to the nearinfrared observing bands as it crossed a large expanse of the Universe, revealed a host galaxy. Gamma-rays are not easily absorbed, unlike lower energy radiation, and travel across the Universe from GRBs pinpointing the locations of star forming galaxies in all directions. This is a unique method of selecting galaxy samples which is complementary to the more established deep surveys of specific regions of sky.

\section{What next?}

The future for GRB science looks bright. The dedicated efforts of the Swift satellite - the fastest satellite in orbit - are bearing fruit. The large number of GRBs being detected and followed up is generating meaningful statistical samples to work with. A large number of key individual sources with excellent datasets continue both to answer existing questions and to raise new ones. The next generation of spaceand ground-based telescopes promise to push even deeper into the darkness, to improve the clarity with which we see the Universe around us, to observe in new windows (very low frequency radio waves, very high energy gamma-rays, neutrino and gravitational wave detection) and to provide ever faster responses to GRB triggers.

Article submitted to Royal Society 


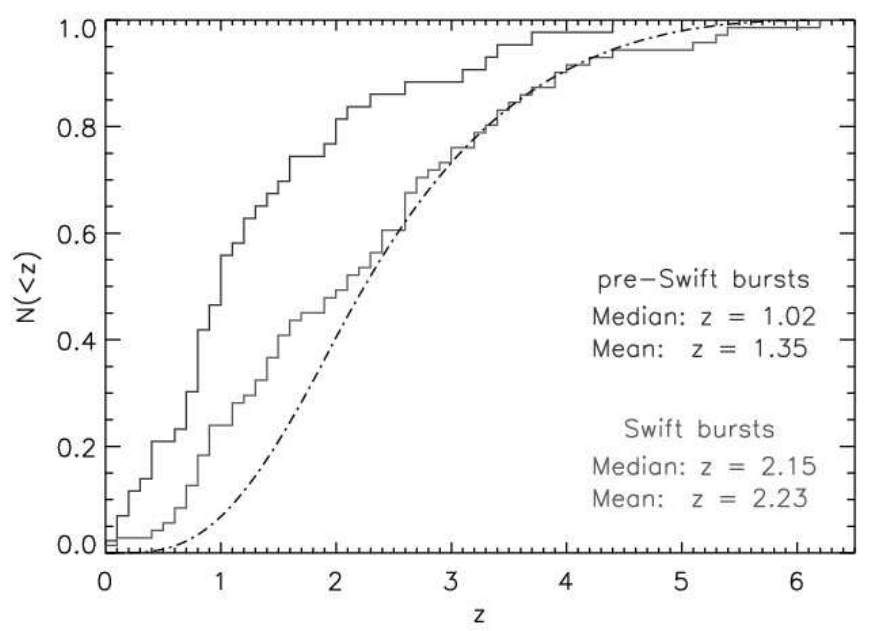

Figure 5. The redshift distribution for pre-Swift (upper cumulative histogram) and Swift (lower histogram) GRBs. GRBs span a very wide range of redshifts and can be seen currently out to $z=6.3$. The dashed line represents a model for the star formation history of the Universe: long GRB origins in massive stars mean that a link is expected between the two curves. Adapted from Jakobsson et al. (2004) and updated 13 June 2008.

RLCS acknowledges support from STFC for the Swift project at the University of Leicester.

\section{References}

Burrows, D. N. et al. 2005 Bright X-ray Flares in Gamma-Ray Burst Afterglows. Science 309 1833-1835.

Campana, S. et al. 2006 The association of GRB 060218 with a supernova and the evolution of the shock wave. Nature 442 1008-1010.

Falcone, A. D. et al. 2006 The Giant X-Ray Flare of GRB 050502B: Evidence for LateTime Internal Engine Activity. ApJ 641 1010-1017.

Fox, D. B. et al. 2005 The afterglow of GRB 050709 and the nature of the short-hard $\gamma$-ray bursts. Nature 437 845-850.

Fruchter, A. S. 2006 Long $\gamma$-ray bursts and core-collapse supernovae have different environments. Nature 441 463-468.

Fynbo, J. P. U. et al. 2006 No supernovae associated with two long-duration $\gamma$-ray bursts. Nature 444 1047-1049.

Galama, T. J. 1998 An unusual supernova in the error box of the $\gamma$-ray burst of 25 April 1998. Nature 395 670-672.

Gehrels, N. et al. 2004 The Swift gamma-ray burst mission. ApJ 611 1005-1020.

Gehrels, N. et al. 2005 A short $\gamma$-ray burst apparently associated with an elliptical galaxy at redshift $z=0.225$. Nature 437 851-854.

Grupe, D. et al. 2007 Swift and XMM-Newton Observations of the Extraordinary GammaRay Burst 060729: More than 125 Days of X-Ray Afterglow. ApJ 662 443-458.

Hjorth, J. et al. 2003 A very energetic supernova associated with the $\gamma$-ray burst of 29 March 2003. Nature 423 847-850.

Hjorth, J. et al. 2005a GRB 050509B: Constraints on Short Gamma-Ray Burst Models. ApJ 630 L117-120. 
Hjorth, J. et al. 2005b The optical afterglow of the short $\gamma$-ray burst GRB 050709. Nature 437 859-861.

Jakobsson, P. et al. A mean redshift of 2.8 for Swift gamma-ray bursts. A\& A 447 897-903.

Kawai, N. et al. 2006 An optical spectrum of the afterglow of a $\gamma$-ray burst at a redshift of $z=6.295$. Nature 440 184-186.

Klebesadel, R. W., Strong I. B. \& Olson R. A. 1973 Observations of Gamma-Ray Bursts of Cosmic Origin. ApJ 182 85-88.

Kouveliotou, C., Meegan, C. A., Fishman, G. J., Bhat, N. P., Briggs, M. S., Koshut, T. M., Paciesas, W. S., Pendleton, G. N. 1993 Identification of two classes of gamma-ray bursts. ApJ 413 L101-104.

MacFadyen, A. I. \& Woosley, S. E. 1999 Collapsars: Gamma-Ray Bursts and Explosions in "Failed Supernovae". ApJ $\mathbf{5 2 4}$ 262-289.

Mazzali, P. A. et al. 2006 A neutron-star-driven X-ray flash associated with supernova SN 2006aj. Nature 442 1018-1020.

Metzger, M. R., Djorgovski, S. G., Kulkarni, S. R., Steidel, C. C., Adelberger, K. L., Frail, D. A., Costa, E., Frontera, F. 1997 Spectral constraints on the redshift of the optical counterpart to the $\gamma$-ray burst of 8 May 1997. Nature 387 878-880.

Nakar, E. 2007 Short-hard gamma-ray bursts. PhR 442 166-236.

Nousek, J.A. et al. 2006 Evidence for a Canonical Gamma-Ray Burst Afterglow Light Curve in the Swift XRT Data. ApJ 642 389-400.

O'Brien, P. T. et al. 2006, The early X-ray emission from GRBs. ApJ 647 1213-1237.

Paczyński, B. 1995 How Far Away Are Gamma-Ray Bursters? PASP 107 1167-1175.

Palmer, D. M. 2005 A giant $\gamma$-ray flare from the magnetar SGR 1806-20. Nature 434 1107-1109.

Pian, E. et al. 2006 An optical supernova associated with the X-ray flash XRF 060218. Nature 442 1011-1013.

Racusin, J. et al. 2008 GRB 080319B: A Naked-Eye Stellar Blast from the Distant Universe. Nature submitted, arXiv:0805.1557.

Rhoads, J. E. 1997 How to Tell a Jet from a Balloon: A Proposed Test for Beaming in Gamma-Ray Bursts. ApJ 487 L1-4.

Sakamoto, T. et al. 2006 Are Short GRBs Really Hard? Proceedings of 'Gamma Ray Bursts in the Swift Era' Eds. S. Holt, N. Gehrels \& J. Nousek, AIP836 43-47.

Soderberg, A. M. et al. 2008 An extremely luminous X-ray outburst at the birth of a supernova. Nature $\mathbf{4 5 3}$ 469-474.

Stanek, K. Z. et al. 2003 Spectroscopic Discovery of the Supernova 2003dh Associated with GRB 030329. ApJ 591 L17-20.

Starling, R. L. C. 2005 Gas and dust properties in the afterglow spectra of GRB 050730. $A \& A 442$ L21-24.

Starling, R. L. C. et al. 2008 Swift captures the spectrally evolving prompt emission of GRB 070616. MNRAS 384 504-514.

Tanvir, N. R., Chapman, R., Levan, A. J. \& Priddey, R. S. 2005 An origin in the local Universe for some short $\gamma$-ray bursts. Nature 438 991-993.

Vreeswijk, P. M. et al. 2007 Rapid-response mode VLT/UVES spectroscopy of GRB 060418. Conclusive evidence for UV pumping from the time evolution of Fe II and Ni II excited- and metastable-level populations. A $\& A 468$ 83-96.

Woosley, S.E. \& Heger, A. 2006 The Progenitor Stars of Gamma-Ray Bursts. ApJ 637 914-921.

Yoon. S.-C. \& Langer, N. 2005 Evolution of rapidly rotating metal-poor massive stars towards gamma-ray bursts. A\&SA 443 643-648.

Zhang, B. \& Mészáros, P. 2004 Gamma-Ray Bursts: progress, problems \& prospects. IJMPA A19 2385-2472. 


\title{
7. AUTHOR PROFILE
}

\author{
Rhaana L.C. Starling
}

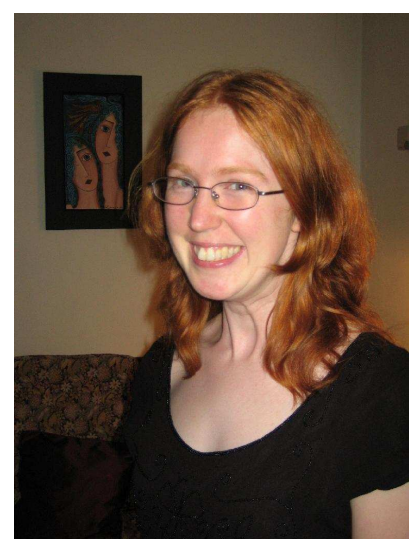

Rhaana studied at St. Andrews University for an MSci Astrophysics (1999) before working as a research assistant at the Harvard-Smithsonian Center for Astrophysics, USA. She returned to the UK for a $\mathrm{PhD}$ in the Central Engines of Active Galactic Nuclei, awarded by University College London in 2004. Rhaana then moved into the field of Gamma-ray Bursts as an EU postdoctoral research associate at the University of Amsterdam, the Netherlands from 2003-2006 and is now a scientist for the Swift satellite mission at the University of Leicester (home of the UK Swift Science Data Centre).

Her research spans a wide range of transient and variable astrophysical objects, with a focus on Gamma-ray Bursts, utilising broad-band observations in the nearinfrared, optical, ultraviolet, X-ray and $\gamma$-ray regimes. 